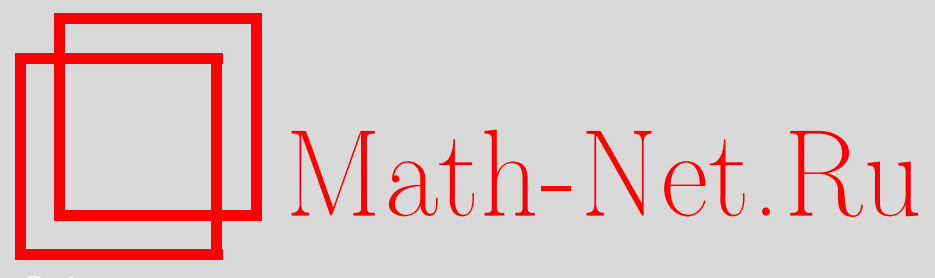

А. Блинков, А. Грибалко, Замкнутые самопересекающиеся ломаные, Квант, 2019, номер 10, 26-28

DOI: https://doi.org/10.4213/kvant20191002

Использование Общероссийского математического портала Math-Net.Ru подразумевает, что вы прочитали и согласны с пользовательским соглашением http://www . mathnet.ru/rus/agreement

Параметры загрузки:

IP: 54.198 .55 .26

26 апреля 2023 г., 17:40:14

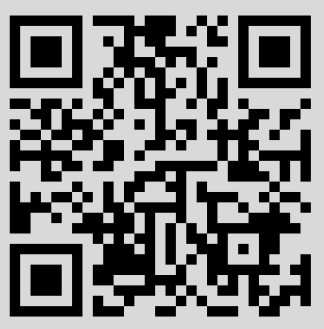




\section{Замкнутые самопересекающиеся ломаные}

\section{А.БЛИНКОВ, А.ГРИБАЛКО}

ЭТОЙ СТАТЬЕ ПОЙДЕТ РЕЧЬ О ЛОМА-

ных линиях на плоскости. Напомним, что, для того чтобы изобразить ломаную, достаточно выбрать несколько точек (не меньше трех), занумеровать их в какомнибудь порядке, после чего последовательно соединить отрезками точки с сосед-

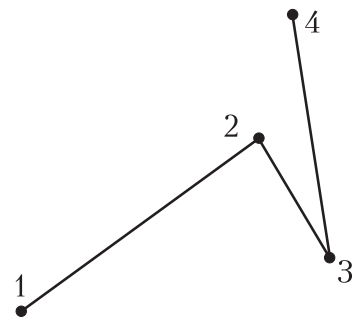

Pис. 1 ними номерами.

Выбранные точки называются вершинами ломаной, а отрезки - ее звеньями (на рисунке 1 трехзвенная ломаная, у которой четыре вершины).

Если хотя бы два звена ломаной пересекаются (в своих внутренних точках), то ее называют самопересекающейся (на рисунке 2 - четырехзвенная самопересекающаяся ломаная).

И наконец, если совместить первую и последнюю вершины ломаной, то ее назы-

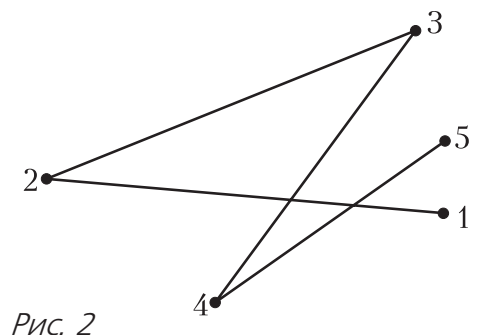

вают замкнутой. В такой ломаной количество вершин совпадает с количеством звеньев (на рисунке 3 - пятизвенная замкнутая ломаная).

Нас прежде всего будут интересовать замкнутые самопересекающиеся ломаные.

Статья была опубликована в журнале «Квантик» №11 за 2019 год.

DOI: https://doi.org/10.4213/kvant20191002

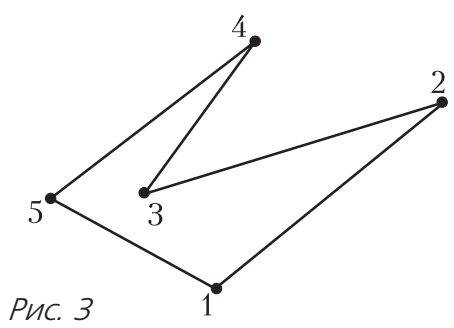

Начнем с задачи, предложенной А.Пешниным (ее частные случаи были использованы на XXV Турнире математических боев имени А.П.Савина).

Задача 1. Сколько вериин может быть у замкнутой ломаной, которая каждое свое звено пересекает ровно два раза?

Решение. Очевидно, что трехзвенная замкнутая ломаная не может быть самопересекающейся. Замкнутая ломаная с четырьмя вершинами также не удовлетворяет условию задачи, так как соседние звенья пересечься не могут, а для каждого звена есть только одно, не являющееся соседним. Пример пятизвенной ломаной хорошо известен - это пятиконечная звезда. Можно, хотя и не обязательно, изобразить такую ломаную так, чтобы длины всех звеньев были одинаковыми (рис.4, $a$; вершины ломаной делят окружность на пять равных частей).

Отметим, что и в данном случае, и в дальнейшем использовать окружность не обязательно, но удобно.
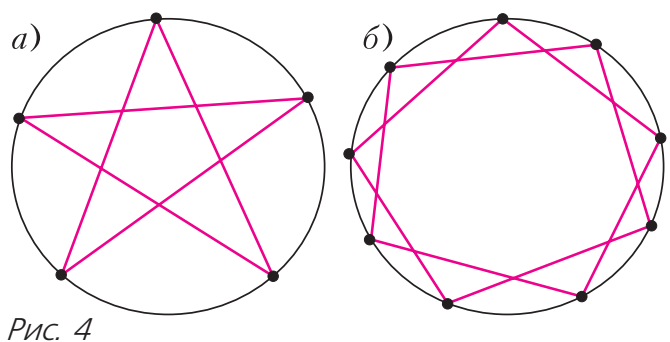

Рис. 4 
Этот пример подсказывает, что аналогичным образом можно построить любую ломаную, удовлетворяющую условию, у которой нечетное количество звеньев, большее трех. Действительно, достаточно поставить на окружности требуемое количество вершин и последовательно соединить их через одну. Например, на рисунке 4,6 изображена ломаная, у которой девять звеньев.

Таким образом, осталось разобраться с ломаными с четным количеством звеньев, начиная с шести.

Искомой шестизвенной ломаной не существует, но доказать это можно только перебором всех случаев, который не очень интересен.

Для восьми звеньев существует красивый пример (рис.5,a). Аналогично можно построить ломаную, удовлетворяющую
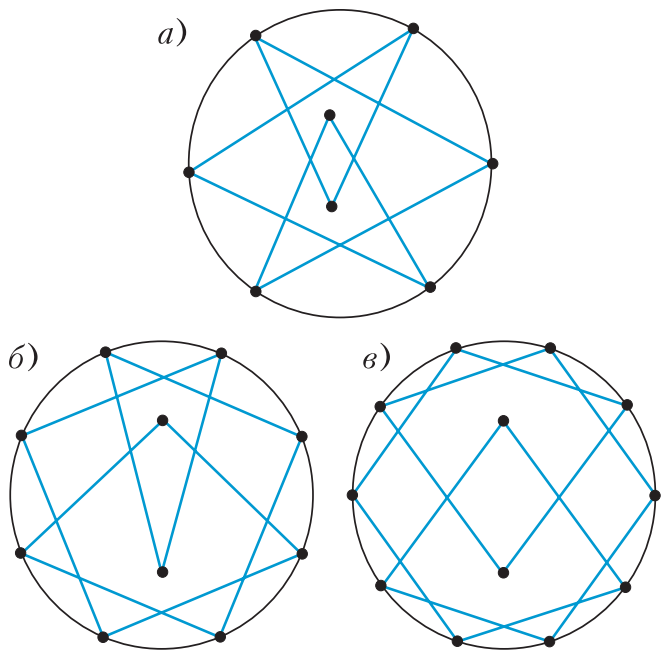

Pис. 5

условию, с любым четным количеством звеньев, большим восьми. Как это делается, понятно из примеров для десяти и двенадцати звеньев, показанных на рисунках 5,б и 5, в. Сначала мы отмечаем на окружности точки, которых на две меньше, чем нужно, и соединяем их через одну. Так как точек четное количество, то получатся две замкнутые ломаные, все звенья которых пересекаются с другой ломаной в двух точках. После этого удаляем по одному звену в каждой ломаной и соединяем ломаные в одну, используя еще две вершины, расположенные внутри окружности.

Однако есть и более простой способ. Воспользуемся тем, что любое четное число, большее восьми, можно представить в виде суммы двух нечетных слагаемых, каждое из которых не меньше пяти.

Покажем, например, как построить двенадцатизвенную ломаную, удовлетворяющую условию. Изобразим две окружности, которые касаются внешним образом в некоторой точке. В одной из окружностей построим уже указанным способом пятизвенную ломаную, а в другой - семизвенную, причем точка касания должна быть их общей вершиной. А теперь эту точку «раздвоим» (рис.6; результат раздвоения - вершины с номерами 1 и 6).

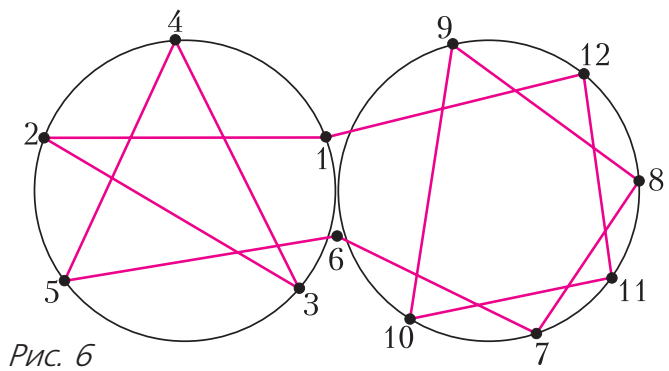

Аналогично строятся все искомые ломаные, у которых количество звеньев четное и больше восьми.

Возникает вопрос: почему мы начали с двух точек пересечения звеньев, а не с одной, что, казалось бы, более естественно?

Дело в том, что такой порядок более логичен, так как решение следующей задачи будет во многом опираться на решение рассмотренной.

Задача 2. Сколько вериин может быть у замкнутой ломаной, которая каждое свое звено пересекает ровно один раз?

Решение. Сразу заметим, что в этом случае звенья ломаной должны разбиваться на непересекающиеся пары, поэтому у искомых ломаных четное количество звеньев. Легко проверить, что замкнутая ломаная из четырех звеньев условию не удовлетворяет.

Пример искомой ломаной из шести звеньев можно построить, исходя из следую- 
щих соображений: помимо того, что не могут пересекаться соседние звенья, не могут пересекаться и звенья, стоящие через одно. Действительно, в этом случае образуется треугольник (рис.7,a), в который можно будет только «войти», если пересечь среднее звено, но нельзя будет «выйти». Поэтому надо пересекать первое звено с четвертым, второе - с пятым и третье с шестым (рис.7,б).
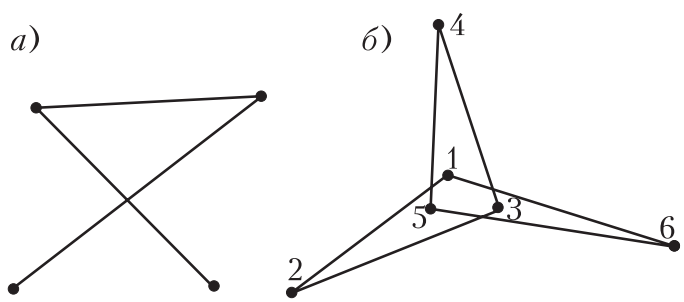

Pис. 7

Пример искомой ломаной из восьми звеньев читателю предлагается построить самостоятельно (см. задачи в конце статьи). А вот пример десятизвенной ломаной можно получить, обратившись к задаче 1. Действительно, рассмотрим пример замкнутой пятизвенной ломаной, которая каждое свое звено пересекает два раза (см. рис.4). «Сломаем» каждое звено между

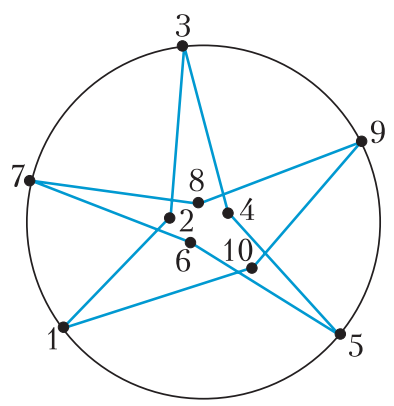

Pnc. 8 двумя точками пересечения и получим искомый пример (рис.8). Аналогично, рассмотрев семизвенную ломаную из задачи 1, можно получить решение для ломаной, у которой 14 звеньев; из примера восьмизвенной ломаной из задачи 1 получим решение для ломаной из 16 звеньев и т.д.

Этот прием не годится только для построения двенадцатизвенной ломаной, так как примера шестизвенной ломаной, которая каждое свое звено пересекает два раза, не существует. Но в этом случае можно использовать другую идею решения задачи 1: «раздвоение». Построим две лома- ные из рисунка 7,б с общей вершиной и «раздвоим» ее (рис.9; результат раздвоения - вершины с номерами 6 и 12). Понятно, что идея «раздвоения» вершин

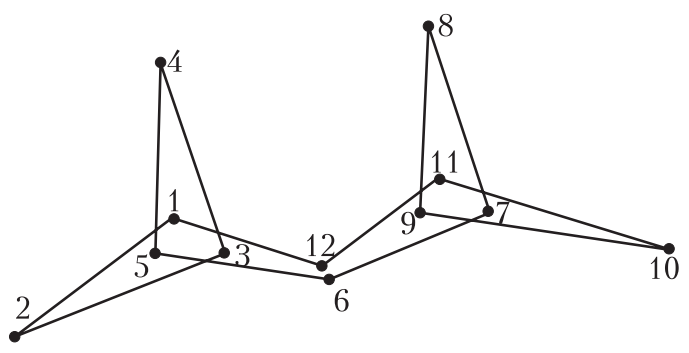

Pnc. 9

более универсальна. В том числе и потому, что позволяет комбинировать ломаные с разным количеством звеньев.

Надеемся, что идеи и приемы, описанные выше, помогут при решении других задач.

\section{Задачи для самостоятельного решения}

1. Может ли прямая, не содержащая вершин замкнутой девятизвенной ломаной, пересечь каждое ее звено?

2 (В.Произволов). Замкнутая ломаная такова, что каждые два ее не соседних звена пересекаются. Докажите, что у этой ломаной нечетное количество звеньев.

3. Существует ли пятнадцатизвенная ломаная, пересекающая каждое свое звено ровно три раза?

4. Постройте восьмизвенную замкнутую ломаную, которая каждое свое звено пересекает один раз.

5 (Д.Калинин, вариация фольклора). Маша нарисовала замкнутую семизвенную ломаную. Для каждого звена она записала, со сколькими звеньями оно пересекается во внутренних точках. Могла ли она записать в каком-нибудь порядке числа 1, 2, 3, 4, 3, 2, 1?

6. Какое наибольшее количество точек самопересечения может иметь замкнутая ломаная, в которой 7 звеньев?

7 (Н.Васильев). Рассматриваются всевозможные шестизвенные замкнутые ломаные, все вершины которых лежат на окружности.

a) Нарисуйте такую ломаную, которая имеет наибольшее возможное количество точек самопересечения.

б) Докажите, что большего количества самопересечений такая ломаная иметь не может. 